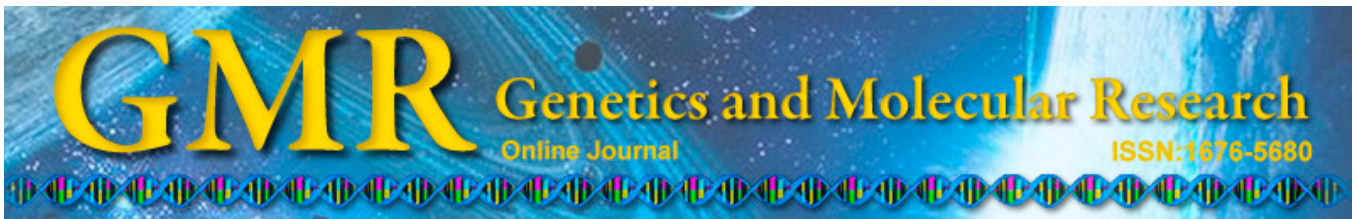

\title{
Expression and clinical significance of PIWIL2 in hilar cholangiocarcinoma tissues and cell lines
}

\author{
Y.J. Chen ${ }^{1}$, X.F. Xiong ${ }^{1}$, S.Q. Wen ${ }^{2}$, L. Tian ${ }^{1}$, W.L. Cheng ${ }^{1}$ and Y.Q. Qi ${ }^{1}$ \\ ${ }^{1}$ Department of Pancreatic-Biliary Surgery, Tongji Hospital, \\ Tongji Medical College, Huazhong University of Science and Technology, \\ Wuhan, Hubei, China \\ ${ }^{2}$ Abdominal Surgery, Puai Hospital, Tongji Medical College, \\ Huazhong University of Science and Technology, Wuhan, Hubei, China \\ Corresponding author: Y.J. Chen \\ E-mail: chenyongjun_1@163.com
}

Genet. Mol. Res. 14 (2): 7053-7061 (2015)

Received October 21, 2014

Accepted January 29, 2015

Published June 26, 2015

DOI http://dx.doi.org/10.4238/2015.June.26.15

\begin{abstract}
The objective of this study was to explore the relationship between PIWI-like protein 2 (PIWIL2) and clinicopathological characteristics and prognosis after radical resection. To accomplish this, we analyzed PIWIL2 expression in hilar cholangiocarcinoma tissues and cell lines. PIWIL2 expression was detected by immunohistochemistry in 41 hilar cholangiocarcinoma samples and 10 control tissues. Western blotting and immunocytofluorescence were used to investigate PIWIL2 expression in the cholangiocarcinoma cell line QBC939 and the bile duct epithelial cell line HIBEpic. Univariate and multivariate survival analyses were performed using the Kaplan-Meier method for hilar cholangiocarcinoma patients who underwent radical resection. PIWIL2 expression was significantly higher in the hilar cholangiocarcinoma tissues and QBC939 cells than in control tissues and HIBEpic cells, respectively $(\mathrm{P}<0.05)$. Poorly and moderately differentiated cholan-
\end{abstract}


giocarcinoma tissues had significantly higher PIWIL2 expression than well-differentiated tissues $(\mathrm{P}<0.05)$. Univariate analysis demonstrated that high PIWIL2 expression was associated with shorter survival time after radical resection $(\mathrm{P}<0.05)$. Multivariate analysis showed that PIWIL2 expression was an independent prognostic factor after radical resection of hilar cholangiocarcinoma $(\mathrm{P}<0.05)$. PIWIL2 expression was also associated with tumor-node-metastasis stage and differentiation. PIWIL2 was an independent prognostic factor after radical resection of hilar cholangiocarcinoma.

Key words: Hilar cholangiocarcinoma; PIWI-like protein 2; Prognosis

\section{INTRODUCTION}

Hilar cholangiocarcinoma (HCCA), also known as Altemeier-Klatskin tumor, is a complex and potentially aggressive disease because of its anatomical position. Because of a lack of efficient early diagnosis indexes, patients often have advanced-stage disease by the time symptoms emerge. Furthermore, the 5-year survival rate of these patients after radical operations is extremely low, as they are often insensitive to chemoradiotherapy (Huang, 2010).

PIWI proteins can silence transposons and other genes, as well as self-genetic elements at the epigenetic and posttranscriptional level. PIWI proteins play important roles in stem cell self-renewal, spermatogenesis, RNA interference, and translation regulation (Lee et al., 2006). A recent study reported that PIWI proteins were only expressed in tumors, and this expression was associated with tumor generation and development; this may be an important new index for tumor diagnosis and prognosis (Lingel and Sattler, 2005). The current study analyzed PIWI-like protein 2 (PIWIL2) expression in HCCA tissues and cell lines in order to determine the relationship between PIWIL2 and clinicopathological factors. This study explored the relationship between PIWIL2 and HCCA.

\section{MATERIAL AND METHODS}

\section{Specimen source}

We selected tissue specimens from 41 patients at Tongji Medical College of Huazhong University of Science and Technology who underwent radical resection of HCCA between February 2007 and March 2012. The patients included 15 females and 26 males with ages ranging from 27 to 76 years (average $=62.7$ years). We also obtained 10 normal bile duct tissue samples from patients with benign biliary tract diseases as control tissues. No patients received chemoradiotherapy before or after surgery, and all patients were followed up for 7 to 48 months after surgery (average $=26.2$ months). No patients withdrew from the study.

\section{Cell culture}

The QBC939 cell line was graciously provided by Professor Wang from the Southwest Hospital Affiliated to the Third Military Medical University. HIBEpic cells were purchased from the CAS cell bank. All cells were cultured in RPMI-1640 media containing 10\% fetal bovine serum. 


\section{Immunohistochemistry}

A rabbit polyclonal antibody for PIWIL2, a SV-0001 2-step Immunostain SP Kit, a DAB color development kit, and an HE Staining Kit were provided by Wuhan Boster Biological Technology, Ltd. (Wuhan, China). Samples were processed according to the instructions in the immunohistochemical kit, with the PIWIL2 antibody diluted 1:100. Stomach cancer sections known to be positive were used as positive controls, and phosphate-buffered saline was used in place of the primary antibody as a negative control. The standard for PIWIL2 positivity was the appearance of brown-yellow or brown granules in the cytoplasm. Scoring of the positivity rate was as follows: 0 points, $<5 \%$ positive; 1 point, 6 to $35 \%$ positive; 2 points, 36 to $70 \%$ positive; 3 points, $>70 \%$ positive. Scoring of staining intensity was as follows: 0 points, no staining; 1 point, light yellow staining; 2 points, brown-yellow staining; 3 points, medium brown staining. The sum of the 2 scoring systems was calculated for an overall score; the low expression group included tissues with overall scores $<4$ points, and the high expression group included tissues with overall scores $\geq 4$ points. We randomly chose 10 high-power fields for observation of each section (less than 10 high power fields were counted according to the actual observed field).

\section{Western blotting}

QBC939 and HIBEpic cells were cultured until they reached 80\% confluence. Cells were then lysed, total protein was extracted, the protein concentration was determined, and proteins were examined by western blotting. Monoclonal antibodies and a polyclonal PIWIL2 antibody were diluted 1:200. The molecular weight of the target band and the optical density were analyzed using gel documentation systems. All tests were repeated 3 times.

\section{Immunofluorescence staining}

Monolayer cells in the logarithmic growth phase were made into a single cell suspension and inoculated onto 6 -well plates covered by a cover glass. When the cell density reached 70 to $80 \%$, cells were fixed with $4 \%$ paraformaldehyde. Cells were then blocked with nonimmune goat serum containing $0.3 \%$ Triton X-100 at room temperature for 60 minutes. The PIWIL2 antibody was added at a 1:100 dilution and incubated overnight at $4{ }^{\circ} \mathrm{C}$. Goat antirabbit IgG antibody was then added at a 1:200 dilution, nuclei were stained with 50 $\mu \mathrm{L}$ DAPI, and samples were mounted with 10 to $20 \mu \mathrm{L}$ anti-fluorescence quenching seal table.

\section{Statistical analysis}

Data were analyzed using the SPSS software, version 19.0 (IBM Corp., Armonk, NY, USA). Comparisons were made using $t$-tests, counting card information using the test. The Kaplan-Meier method was used for univariate survival analysis, and the results were tested using log-rank tests. $\mathrm{P}<0.05$ was considered to be statistically significant.

\section{RESULTS}

\section{PIWIL2 expression in HCCA}

PIWIL2 expression was detected by immunohistochemistry in 41 HCCA samples. 
There were 33 cases $(80.5 \%)$ with high expression (Figure 1A-D) and 8 cases $(19.5 \%)$ with low expression. Among 10 control tissues, 9 cases (90\%) had low or negative expression. Expression of PIWIL2 was higher in HCCA than in normal tissue; this difference was statistically significant $\left(\chi^{2}=17.97, \mathrm{P}<0.05\right.$; Table 1$)$.
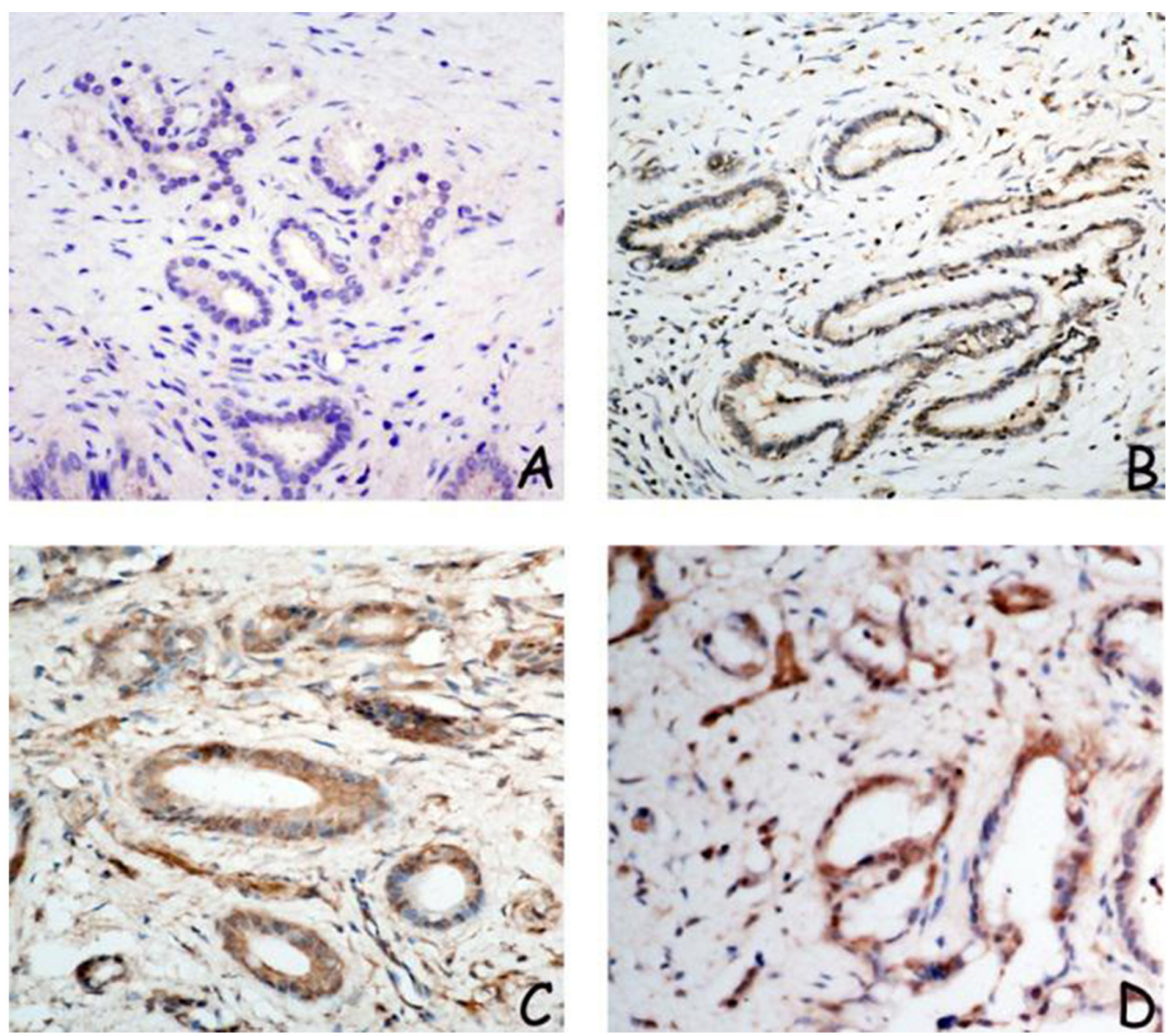

Figure 1. Expression of PIWIL2 in normal bile duct tissue (A), well-differentiated adenocarcinoma (B), moderately differentiated adenocarcinoma $(\mathbf{C})$, and poorly differentiated adenocarcinoma (D).

Table 1. Expression of PIWIL2 in hilar cholangiocarcinoma.

\begin{tabular}{lcccc}
\hline Group & Cases & Low PIWIL2 expression & High PIWIL2 expression & P \\
\hline Adenocarcinoma & 41 & 8 & 33 & $<0.05$ \\
Normal bile duct tissue & 10 & 9 & 1 & \\
\hline
\end{tabular}




\section{Association between PIWIL2 and the degree of HCCA differentiation}

Among the 41 HCCA specimens, 35 cases $(85.5 \%)$ were adenocarcinoma and 6 cases $(14.5 \%)$ were non-adenocarcinoma. Of the 9 well-differentiated adenocarcinomas, $4(44.4 \%)$ had high expression of PIWIL2. Of the 14 moderately differentiated adenocarcinomas, $12(85.7 \%)$ had high expression of PIWIL2. Of the 12 poorly differentiated adenocarcinomas, $11(91.6 \%)$ had high expression of PIWIL2. Statistical analysis showed no difference between PIWIL2 expression in moderately versus poorly differentiated adenocarcinoma $\left(\chi^{2}=0.224, \mathrm{P}=0.636\right)$. However, the well-differentiated group had significantly higher PIWIL2 expression than the moderately differentiated group $\left(\chi^{2}=4.407, \mathrm{P}<0.05\right)$ and the poorly differentiated group $\left(\chi^{2}=5.61, \mathrm{P}<0.05\right)$ (Table 2, Figure $\left.1 \mathrm{~A}-\mathrm{D}\right)$.

Table 2. Relationship between PIWIL2 expression and the degree of differentiation of hilar cholangiocarcinoma.

\begin{tabular}{lccccc}
\hline Differentiation degree & Cases & Low PIWIL2 expression & High PIWIL2 expression & \% high expression & P \\
\hline Well-differentiated & 9 & 5 & 4 & $44.4 \%$ & \\
Moderately-differentiated & 14 & 2 & 12 & $85.7 \%$ & $<0.05^{1}$ \\
Poorly-differentiated & 12 & 1 & 11 & $91.6 \%$ & $<0.05^{2}$ \\
\hline
\end{tabular}

All were pathologically classified as adenocarcinoma; ${ }^{1}$ Comparison between the highly differentiated versus moderately differentiated groups; ${ }^{2}$ Comparison between the highly differentiated versus poorly differentiated groups.

\section{PIWIL2 expression in QBC939 and HIBEpic cells}

Western blotting revealed that expression of PIWIL2 was significantly higher in QBC939 cells than in HIBEpic cells $(\mathrm{P}<0.05)$, as shown by specific bands located at 110 $\mathrm{kDa}$ (Figure 2A and B). Immunofluorescence showed specific expression of green fluorescent PIWIL2 in the cytoplasm of QBC939 cells after excitation. Consistent with our western blot results, the fluorescence intensity was significantly higher in the QBC939 cells than in the HIBEpic cells (Figure 3A and B).
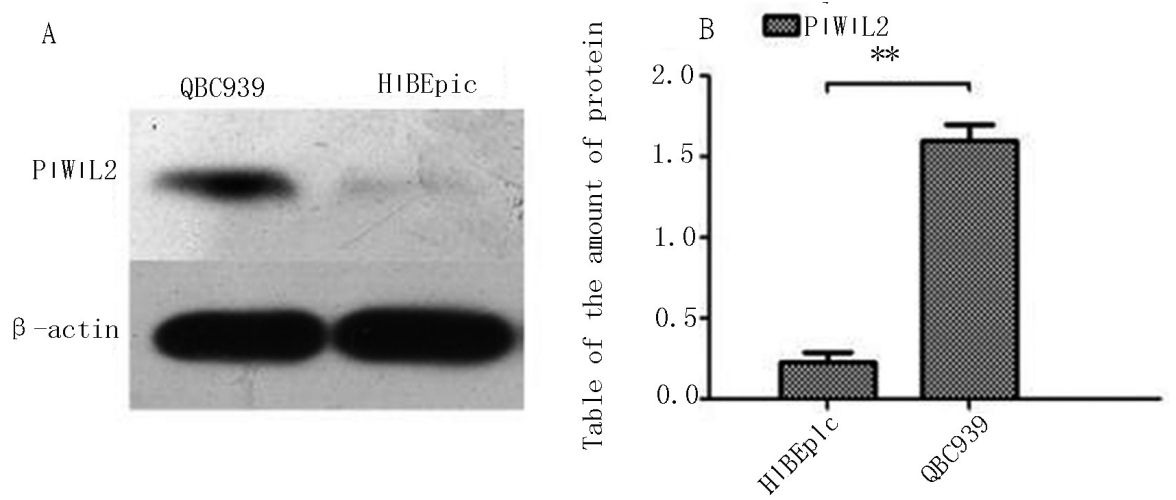

Figure 2. Expression of PIWIL2 in QBC939 and HIBEpic cells detected by western blot. 

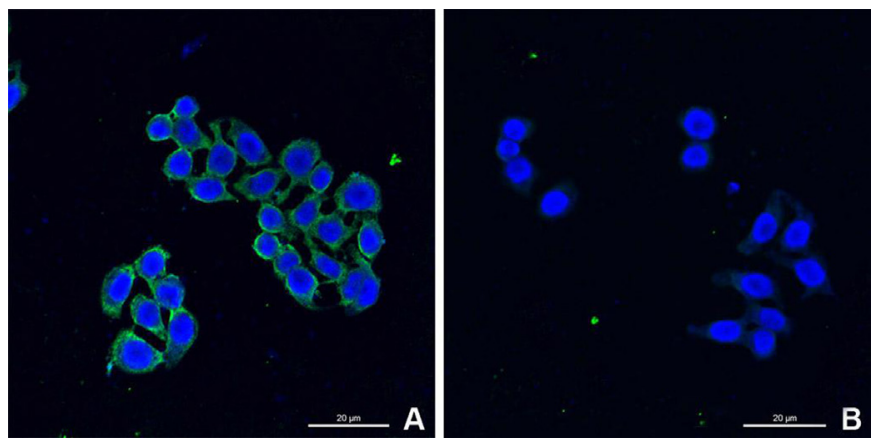

Figure 3. Expression of PIWIL2 in A) QBC939 cells and B) HIBEpic cells detected by immunocytochemistry.

\section{Relationship between PIWIL2 and pathologic factors}

High PIWIL2 expression was not related to age, gender, tumor size, pathologic type, or lymphatic metastasis $(\mathrm{P}>0.05)$. High PIWIL2 expression differed significantly according to tumor-node-metastasis (TNM) stage and degree of differentiation $(\mathrm{P}<0.05)$; high PIWIL2 expression was significantly associated with poor differentiation and high TNM stage (Table 3 ).

Table 3. Relationship between PIWIL2 and clinicopathological characteristics of hilar cholangiocarcinoma.

\begin{tabular}{|c|c|c|c|c|c|}
\hline Pathological feature & Cases & Low PIWIL2 expression & High PIWIL2 expression & $\%$ high expression & $\mathrm{P}$ \\
\hline \multicolumn{6}{|l|}{ Age (years) } \\
\hline$<60$ & 14 & 4 & 10 & $71.4 \%$ & \multirow[t]{2}{*}{0.292} \\
\hline$\geq 60$ & 27 & 4 & 23 & $85.2 \%$ & \\
\hline \multicolumn{6}{|l|}{ Gender } \\
\hline Female & 15 & 5 & 10 & $66.6 \%$ & \multirow[t]{2}{*}{0.090} \\
\hline Male & 26 & 3 & 23 & $88.5 \%$ & \\
\hline \multicolumn{6}{|l|}{ Tumor size $(\mathrm{cm})$} \\
\hline$<3$ & 16 & 2 & 14 & $87.5 \%$ & \multirow{2}{*}{0.365} \\
\hline$\geq 3$ & 25 & 6 & 19 & $76.0 \%$ & \\
\hline \multicolumn{6}{|l|}{ Pathologic type } \\
\hline Non-adenocarcinoma & 4 & 2 & 2 & $50.0 \%$ & \multirow[t]{2}{*}{0.105} \\
\hline Adenocarcinoma & 37 & 6 & 31 & $83.8 \%$ & \\
\hline \multicolumn{6}{|l|}{ Differentiation degree } \\
\hline Well-differentiated & 11 & 5 & 6 & $54.5 \%$ & \\
\hline Moderately-differentiated & 15 & 2 & 13 & $86.7 \%$ & 0.068 \\
\hline Poorly-differentiated & 15 & 1 & 14 & $93.3 \%$ & 0.020 \\
\hline \multicolumn{6}{|l|}{ Lymphatic metastasis } \\
\hline Yes & 23 & 6 & 17 & $73.9 \%$ & \multirow[t]{2}{*}{0.230} \\
\hline No & 18 & 2 & 16 & $88.9 \%$ & \\
\hline \multicolumn{6}{|l|}{ TNM stage } \\
\hline I-II & 16 & 6 & 10 & $62.5 \%$ & \multirow[t]{2}{*}{0.020} \\
\hline III-IV & 25 & 2 & 23 & $92.0 \%$ & \\
\hline
\end{tabular}

TNM, tumor-node-metastasis.

\section{PIWIL2 expression and survival analysis of HCCA patients after radical resection}

Survival analysis was conducted using the Kaplan-Meier method and was confirmed using log-rank tests. Our results show that the group with high expression of PIWIL2 had shorter disease-free survival and overall survival than the group with low PIWIL2 expression $(\mathrm{P}<0.05)$ (Table 4, Figure 4A and B). Multivariate analysis revealed that PIWIL2 expression was an independent prognostic factor for patients after radical resection of HCCA (Table 5). 
Table 4. PIWIL2 expression and survival analysis of hilar cholangiocarcinoma patients after radical resection.

\begin{tabular}{|c|c|c|c|c|c|}
\hline Pathological feature & Cases & Overall survival & $\mathrm{P}$ & Disease-free survival & $\mathrm{P}$ \\
\hline \multicolumn{6}{|l|}{ Age (years) } \\
\hline$<60$ & 14 & 22.3 & \multirow[t]{2}{*}{0.753} & 18.4 & \multirow[t]{2}{*}{0.821} \\
\hline$\geq 60$ & 27 & 26.1 & & 24.7 & \\
\hline \multicolumn{6}{|l|}{ Gender } \\
\hline Female & 15 & 29.6 & \multirow[t]{2}{*}{0.832} & 27.1 & \multirow[t]{2}{*}{0.754} \\
\hline Male & 26 & 26.2 & & 23.6 & \\
\hline \multicolumn{6}{|l|}{ Tumor size $(\mathrm{cm})$} \\
\hline$<3$ & 16 & 28.3 & \multirow[t]{2}{*}{0.907} & 23.7 & \multirow[t]{2}{*}{0.921} \\
\hline$\geq 3$ & 25 & 27.8 & & 22.3 & \\
\hline \multicolumn{6}{|l|}{ Differentiation degree } \\
\hline Well-differentiated & 11 & 32.4 & \multirow[t]{3}{*}{0.328} & 29.8 & \multirow[t]{3}{*}{0.275} \\
\hline Moderately-differentiated & 15 & 26.3 & & 23.2 & \\
\hline Poorly-differentiated & 15 & 15.2 & & 12.1 & \\
\hline \multicolumn{6}{|l|}{ PIWIL2 expression } \\
\hline High expression & 33 & 25.3 & \multirow[t]{2}{*}{0.013} & 22.8 & \multirow[t]{2}{*}{0.021} \\
\hline Low expression & 8 & 37.7 & & 33.6 & \\
\hline \multicolumn{6}{|l|}{ Lymphatic metastasis } \\
\hline Yes & 23 & 27.6 & \multirow[t]{2}{*}{0.475} & 23.7 & \multirow[t]{2}{*}{0.327} \\
\hline No & 18 & 25.2 & & 21.5 & \\
\hline \multicolumn{6}{|l|}{ TNM stage } \\
\hline I-II & 16 & 32.1 & \multirow[t]{2}{*}{0.217} & 28.2 & \multirow[t]{2}{*}{0.173} \\
\hline III-IV & 25 & 23.4 & & 20.3 & \\
\hline
\end{tabular}

TNM, tumor-node-metastasis.
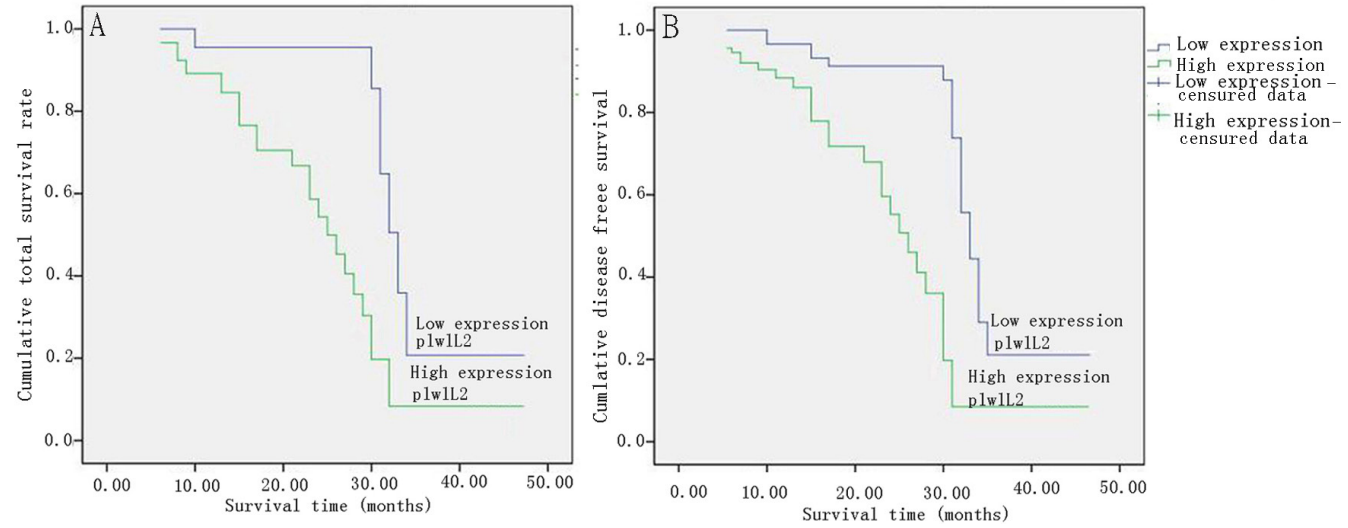

Figure 4 Survival curves for hilar cholangiocarcinoma patients.

Table 5. Multivariate analysis of hilar cholangiocarcinoma after radical resection.

\begin{tabular}{|c|c|c|c|c|c|c|}
\hline \multirow[t]{2}{*}{ Variables } & \multicolumn{3}{|c|}{ Disease-free survival } & \multicolumn{3}{|c|}{ Overall survival } \\
\hline & $\mathrm{P}$ & $95 \% \mathrm{CI}$ & $\mathrm{EXP}(\mathrm{B})$ & $\mathrm{P}$ & $95 \% \mathrm{CI}$ & $\operatorname{EXP}(B)$ \\
\hline Tumor size & 0.723 & $0.431-2.012$ & 0.865 & 0.827 & $0.475-2.103$ & 0.954 \\
\hline Differentiation degree & 0.815 & $0.528-1.836$ & 0.931 & 0.869 & $0.507-1.871$ & 0.917 \\
\hline PIWIL2 expression & 0.024 & $0.088-0.916$ & 0.247 & 0.026 & 0.091-0.902 & 0.253 \\
\hline Lymphatic metastasis & 0.514 & $0.342-1.614$ & 0.810 & 0.712 & $0.431-1.792$ & 0.873 \\
\hline TNM stage & 0.298 & $0.682-3.861$ & 1.497 & 0.421 & $0.598-3.609$ & 1.536 \\
\hline
\end{tabular}

$\mathrm{CI}$, confidence interval; TNM, tumor-node-metastasis. 


\section{DISCUSSION}

The PIWI protein was first discovered in germline stem cells from Drosophila melanogaster in 1997 as a protein that was mainly expressed in germ cells and interacted with PIWI RNA (Lin and Spradling, 1997). PIWI plays important roles in stem cell self-renewal, RNA silencing, and transcriptional regulation. Currently, 4 PIWI subfamilies have been identified in humans: PIWIL1/HIWI, PIWIL2/HILI, PIWIL3, and PIWIL4/HIWI2 (Carmell et al., 2007). Recent studies have found that the PIWI family member PIWIL2 is involved in tumorigenesis by activating Stat3/Bcl-XL, enhancing apoptosis of Stat3/cyclin D1 signaling pathway Stat3, 5, and upregulating genes encoding cell division inhibitory factors and regulatory factors to stimulate cell proliferation and inhibit cell apoptosis (Buettner et al., 2002). Studies have confirmed that PIWIL2 is closely related to the development of breast cancer and cervical cancer (He et al., 2010; Lu et al., 2012).

In this study, we detected expression of PIWIL2 in HCCA and normal bile duct tissues and found that PIWIL2 was expressed at significantly higher levels in HCCA than in normal bile duct tissues $(\mathrm{P}<0.05)$. We also detected PIWIL2 expression in the cell lines QBC939 and HIBEpic. PIWIL2 expression was significantly higher in QBC939 cells compared to HIBEpic cells $(\mathrm{P}<0.05)$. Further analysis of PIWIL2 expression in HCCA specimens and pathologic factors revealed that high expression of PIWIL2 was significantly associated with poor differentiation ( $\mathrm{P}<0.05)$. Wang et al. (2012) found that expression of HIWI, PIWIL2, PIWIL3, and HIWI2 was positively associated with tumor staging, lymphatic metastasis, and TNM stage; high expression was also associated with shorter survival. These observations are in agreement with our study. Disease-free survival and overall survival were shorter in the group with high PIWIL2 expression compared to the group with low expression $(\mathrm{P}<0.05)$. It is possible that PIWIL2 plays regulatory roles in the formation and development of HCCA as an oncogene, but the mechanism of PIWIL2 action in HCCA needs to be investigated further.

In conclusion, we found that PIWIL2 expression in HCCA was closely associated with the degree of differentiation $(\mathrm{P}<0.05)$, and multivariate analysis showed that PIWIL2 expression was an independent prognostic factor for patients after radical resection of HCCA $(\mathrm{P}<$ 0.05); therefore, PIWIL2 expression may be a diagnostic and prognostic marker for HCCA.

\section{ACKNOWLEDGMENTS}

Research supported by the National Natural Science Foundation (\#81071998, \#81372239) and the Hubei Province Natural Science Fund for Distinguished Young Scholars (\#2012FFA012).

\section{REFERENCES}

Buettner R, Mora LB and Jove R (2002). Activated STAT signaling in human tumors provides novel molecular targets for therapeutic intervention. Clin. Cancer Res. 8: 945-954.

Carmell MA, Girard A, van de Kant HJ, Bourc'his D, et al. (2007). MIWI2 is essential for spermatogenesis and repression of transposons in the mouse male germline. Dev. Cell 12: 503-514.

He G, Chen L, Ye Y, Xiao Y, et al. (2010). Piwil2 expressed in various stages of cervical neoplasia is a potential complementary marker for p16. Am. J. Transl. Res. 2: 156-169.

Huang ZQ (2010). 25 years of surgical treatment on hilar cholangiocarcinoma. Chin. J. Digest. Surg. 3: 161-164.

Lee JH, Schütte D, Wulf G, Füzesi L, et a1. (2006). Stem-cell protein Piwil2 is widely expressed in tumors and inhibits apoptosis through activation of Stat3/Bcl-xL pathway. Hum. Mol. Genet. 15: 201-211. 
Lin H and Spradling AC (1997). A novel group of pumilio mutations affects the asymmetric division of germline stem cells in the Drosophila ovary. Development 124: 2463-2476.

Lingel A and Sattler M (2005). Novel modes of protein-RNA recognition in the RNAi pathway. Curr. Opin. Struct. Biol. 15: 107-115.

Lu Y, Zhang K, Li C, Yao Y, et al. (2012). Piwil2 suppresses p53 by inducing phosphorylation of signal transducer and activator of transcription 3 in tumor cells. PLoS One 7: e30999.

Wang Y, Liu Y, Shen X, Zhang X, et al. (2012). The PIWI protein acts as a predictive marker for human gastric cancer. Int. J. Clin. Exp. Pathol. 5: 315-325. 